\title{
Le nouveau visage de la vieillesse
}

Jean Martin

\footnotetext{
* On pense en particulier à la grande étude épidémiologique CoLaus (pour cohorte lausannoise), initiée sur l'initiative de l'Institut universitaire de médecine sociale et préventive de Lausanne en 2003, qui a apporté beaucoup d'enseignements. Récemment, voir aussi: Sirven N., en collab. avec Th. Rochereau. Mesurer la fragilité des personnes âgées en population générale: une comparaison entre les enquêtes ESPS et SHARE. Paris: IRDES (Institut de recherche et documentation en économie de la santé). Questions d'économie de la santé, juin 2014, № 199.
}

jean.martin[at]saez.ch
Dans la très utile collection «Le savoir suisse» (considérée comme l'homologue des «Que sais-je» des Presses universitaires de France) paraît un livre de deux auteurs de l'Université de Genève, acteurs des travaux des décennies récentes concernant les personnes âgées et la gérontologie. L'ouvrage récapitule les principaux résultats du programme «Swiss Interdisciplinary Longitudinal Study on the Oldest Old» (SWILSOO), qui notamment a suivi deux cohortes d'octogénaires entre 1994 et 2004, ainsi que d'autres études du CIGEV (Centre interfacultaire de gérontologie et d'études des vulnérabilités). On sait que vulnérabilités et processus de fragilisation à l'âge avancé sont aujourd'hui au premier plan des préoccupations de la médecine et des responsables des systèmes sanitaire et social - en rapport avec ce qu'on peut appeler le «tsunami gris», l'augmentation de la proportion des personnes âgées dans la population.

Cet ouvrage est construit, disent ses auteurs, à partir du croisement de deux regards - en une sorte de fructification croisée. D'un côté celui de l'observateur, qui avec ses mesures, ses statistiques et son

\section{Le quatrième âge n'est pas caractérisé par l'état de dépen- dance mais par la fragilité.}

souci d'objectivité analyse la population âgée et ses transformations; de l'autre, le regard des témoins qui racontent leur vieillissement et leur manière d'y faire face (récits et opinions recueillis soit par questionnaire standardisé, soit lors d'entretiens ouverts). Le lecteur voit/vit ainsi l'alternance des données agrégées et graphiques, d'une part, et des transcriptions d'entretiens avec des personnes très âgées d'autre part. Modalité tout à fait intéressante qui facilite l'étude de l'ouvrage, l'«anime».

On sait les grandes mutations démographiques, sociologiques, matérielles qu'a connues dans nos pays le domaine de la vieillesse. Qu'il suffise de rappeler par exemple que, en Suisse, l'âge choisi comme marqueur du passage à la retraite, à savoir 65 ans, correspondait à peu près à l'espérance de vie de l'époque! Alors qu'aujourd'hui le jeune retraité a souvent devant lui une vingtaine d'années à vivre.

L'ouvrage comprend neuf chapitres. Dans le premier, les auteurs discutent le concept même de «quatrième âge», avec des repères historiques et socio-

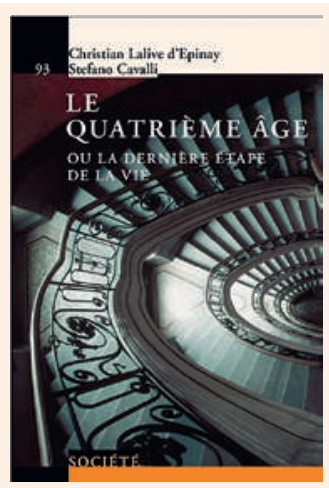

Christian Lalive d'Epinay, Stefano Cavalli Le Quatrième Âge ou la dernière étape de la vie

Lausanne: Presses polytechniques et universitaires romandes - Collection «Le savoir suisse»; 2013. 142 pages.

ISBN 978-2-8891-5048-9

logiques. Ainsi à propos de l'émergence en Amérique du Nord, dès les années 1980, de la distinction entre les young old, les old old, enfin les oldest old. Le chapitre 2 s'efforce de circonscrire les caractéristiques de la vieillesse et des circonstances et trajectoires de santé des vieux, et permet la conclusion suivante: le quatrième âge n'est pas caractérisé par l'état de dépendance mais par la fragilité - cette dernière étant aujourd'hui, logiquement, l'objet de nombreux travaux.*

La conscience d'«être vieux» est le sujet du chapitre 3, constitué pour l'essentiel des propos recueillis auprès d'une dizaine de personnes dont les plus jeunes ont 85 ans. On voit que les vieillards récusent toute définition catégorielle qui recourt comme marqueur à l'âge chronologique. Chez eux, l'éveil de la conscience d'être vieux est lié à l'expérience vécue de leur fragilisation - que la prise de conscience s'opère progressivement ou qu'elle soit la conséquence d'un évènement déclencheur.

\section{Les vieillards récusent toute défini- \\ tion catégorielle qui recourt comme marqueur à l'âge chronologique.}

Le chapitre 4, traitant d'inégalités, fait d'abord une distinction: une différence est un écart entre deux individus ou groupes sur un paramètre donné, sans qu'il y ait a priori jugement de valeur. Cette différence devient une inégalité quand une société l'inscrit dans un système hiérarchique (échelle de pouvoir voire de privilège). Est traité le «paradoxe des genres» pour lequel on n'a pas encore d'explication: 
les femmes vivent plus longtemps que les hommes, mais ces derniers (quand ils deviennent vieux) sont en meilleure santé qu'elles. Les auteurs pointent aussi le fait que, aujourd'hui encore, il subsiste en Suisse des «poches de pauvreté» parmi les vieux et très vieux: notamment chez les petits indépendants (agriculteurs, par exemple en montagne, artisans) qui n'étaient pas soumis obligatoirement au système de pensions dont bénéficie la majorité. Toujours à propos d'inégalités sociales (sujet majeur de la santé publique bien sûr - et de la sociologie), Lalive et Cavalli relèvent: «Si au grand âge la position dans l'échelle sociale n'exerce plus d'incidence sur le temps qui reste à vivre, en revanche elle agit toujours sur les conditions (plus ou moins favorables) dans laquelle ce temps sera vécu.»

Intéressante discussion au chapitre 5 sur la «métamorphose des mondes de vie quotidienne» (alltägliche Lebenswelt): mutations majeures des modes de vie, des horizons physique, temporel, relationnel, professionnel. Entre autres à propos de la douleur et du risque au quotidien. Dans la vie relationnelle, la problématique de la réciprocité (référence à la théorie du don de Marcel Mauss) joue un rôle important. Le don nourrit la relation mais en même temps il crée un rapport de force. «Préserver pour soi un statut de donneur est un enjeu identitaire essentiel tout au long de la vie», y compris dans la vieillesse.

Comment les personnes très âgées font-elles face aux perturbations de la vie quand la réalité et les attentes de la personne divergent («nouvel acte de la dialectique entre nature et culture»)? C'est le sujet du chapitre 6. Deux types de stratégies sont possibles: 1) celles qui tendent à changer la réalité pour la rendre plus conforme aux attentes; 2 ) celles qui reformulent les attentes pour les rendre compatibles avec la réalité. Tirant profit de témoignages (récits de vieillesse), les auteurs distinguent quatre moments principaux dans la résilience dont font preuve les vieux: le constat, l'acceptation (ou le refus), la reconstruction de soi, les aménagements du mode de vie.

\section{La conscience de la continuité du moi jusqu'au terme de} la vie est le fruit d'un travail narratif autobiographique.

Le chapitre 7 traite de la problématique majeure à laquelle sont confrontés une fois ou l'autre la majorité des aînés. Garder son domicile ce qui, petit à petit ou plus rapidement, exige de pouvoir recourir à des aidants (proches dans un premiers temps, puis services à domicile); ou intégrer un établissement médico-social (EMS). Les réactions, réserves, craintes, sont diverses selon les personnes et leur histoire mais l'expérience montre que, le plus souvent, les aînés s'habituent raisonnablement bien - ou très bien - à leur nouveau domicile (collectif). A noter ici des différences notables suivant les parties de la Suisse (et selon le caractère rural ou plus urbanisé de la région), liées en particulier à l'intensité variable du développement des services de soins et d'aide à domicile - en général plus étoffés en Suisse romande. Est présenté ce qu'on a appris des déterminants de la décision d'entrer en EMS, avec ici aussi des témoignages éclairants. Les auteurs relèvent que, pour les personnes concernées, «réinterpréter ce qui s'est passé, dire que l'on a choisi l'EMS alors qu'avant on le récusait, est une manière de récupérer, sur le plan symbolique, le sentiment d'être le sujet de sa propre vie».

Les deux derniers chapitres (8 et 9) abordent des thèmes à connotation philosophique et de sens de la vie: «Rester qui je suis: de l'identité au grand âge», et «Aimer la vie - Accepter la mort». A propos d'identité: c'est le «principe subjectif qui permet à la personne d'entretenir une perception de continuité au cours de sa vie [...] Elle résulte d'un travail que la personne exerce sur elle-même, qui se cristallise sous la forme d'une représentation de soi qui évolue au cours de l'avancée en âge, en même temps que se préserve un noyau dur. Un sprincipe de continuité dans le changement.» Trois récits illustrent comment le travail de mémoire de la personne âgée est un «chantier permanent de reconstruction».

\section{... un ouvrage substantiel, bien écrit, remplissant bien sa «mission»...}

Dans leurs dernières pages, Lalive et Cavalli concluent que la préservation de l'identité, donc la conscience de la continuité du moi jusqu'au terme de la vie, est le fruit d'un travail narratif autobiographique. Et ils parlent de ces «vieillards qui jour après jour s'efforcent de faire triompher la culture sur une nature qui donne des signes d'épuisement, de préserver la vie et de lui donner un sens». Derrière ces efforts, disent-ils, «se profile l'ombre de Sisyphe» qui chaque jour doit à nouveau rouler son rocher au haut de la montagne... Je souligne toutefois que cette remarque ne doit pas faire croire que le ton de l'ouvrage serait pessimiste, ce n'est en rien le cas.

En conclusion, un ouvrage substantiel, bien écrit, remplissant bien sa «mission» de présenter sous forme ramassée un sujet médico-social et de société de grande importance. Lecture recommandée. 